The Israeli Journal of Aquaculture - Bamidgeh, IJA_72.2020.959575, 12 pages

CCBY-NC-ND-4.0・https://doi.org/10.46989/001c.21169

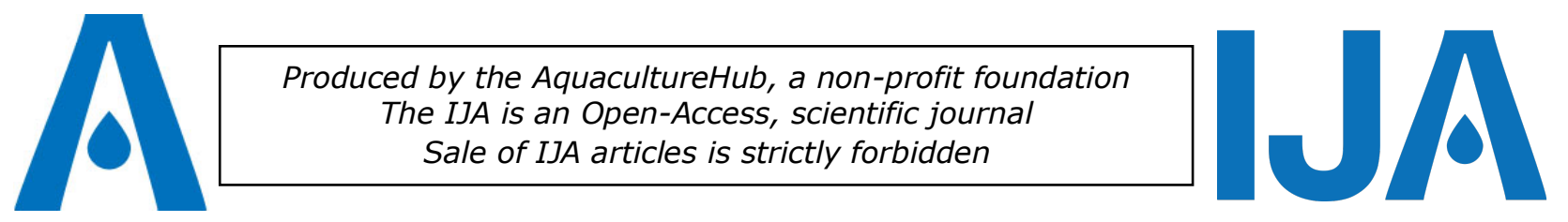

\title{
Revealing the difference of intestinal microbiota composition of cultured European eels (Anguilla anguilla) with different growth rates
}

\author{
Ye Shi", De-Ying Ma*, Shao-Wei Zhai *
}

\begin{abstract}
Fisheries College of Jimei University, Engineering Research Center of the Modern Industry Technology for Eel, Ministry of Education, Xiamen 361021, China
\end{abstract}

Keywords: European eels; growth rate; intestinal microbiota

\begin{abstract}
Autochthonous probiotics were proposed to have a higher capacity to compete with resident pathogens and were also more prone to dominate and persist over other potentially pathogenic microbes. Therefore, the aim of this study was conducted to investigate the potential autochthonous microbiota by revealing the differences in the intestinal microbiota composition of European eels (Anguilla anguilla) with different growth rates. The differences of diversity, an abundance of the intestinal microbiota, and compositions of all the libraries were identified. Moreover, the composition of the intestinal microbiota of European eels was affected by the growth rates. The dominant phylum of the intestinal microbiota of fast growth eels was Fusobacteria, while Spirochaetes was the dominant phylum of the intestinal microbiota of slow growth and medium growth eels. Cetobacterium was identified as the predominant genera of the intestinal microbiota of fast growth eels; there was a significant difference in this genus between fast growth eels and slow growth eels. Although the relative abundances of Plesiomonas, Turicibacter, Nitrospira, and Lachnospiraceae bacterium NK4A136 group were also increased with the growth rates, only Cetobacterium seemed to have great potential as the probiotics to European eels.
\end{abstract}

\footnotetext{
*Corresponding author: Tel: +86592 6181420, Fax: +86592 6181476. e-mail: shaoweizhai@hotmail.com

\# These two authors contributed equally to this work.
} 


\section{Introduction}

The European eel (Anguilla anguilla) is one of the species for eel cultivation in the world. To date, all the seedlings for eel cultivation are wild glass eels or elvers collected in the estuarine waters. While the natural stocks of European eel have decreased markedly due to overfishing, environmental destruction, oceanographic/climatic changes, and other as yet unknown factors during the last several decades (Jacoby et al., 2015), this fish has been included in the Red List of the IUCN, as a Critically Endangered Species. Unfortunately, the application of many measures has not reduced the risk of extinction (Mordenti et al., 2012). One realistic solution to the issue would be to improve the utilization efficiency of wild glass eels.

The eel, just like other fish species in commercial aquaculture, is usually reared in enclosed spaces, and efforts have been made to increase productivity per unit space. There was an elevated risk for disease outbreak and substantial loss for farmers, and several drawbacks were caused by prophylactic administration of antibiotics, strict regulations have been established to ban or minimize their application in aquaculture. As an alternative to antibiotics, dietary administration of probiotics has received increasing attention during the past three decades for control or treatments of bacterial, viral, and parasitic diseases of fish (Hoseinifar et al., 2018). Autochthonous bacteria from fish intestine have been suggested as a primary source of potential probiotics in aquaculture (Mladineo et al., 2016), they might protect against diseases including strengthening immune response, competition for binding sites, production of antibacterial substances, and competition for nutrients (Hoseinifar et al., 2018). Some studies further indicated that bacteria isolated from the intestine of the studied fish might be the best potential probiotics for themselves (Sun et al., 2009; Mladineo et al., 2016; Hoseinifar et al., 2018).

At present, the intestinal microbiota of eels was not well studied, and only several studies were conducted to investigate the intestinal microbiota of different eel species, including the mucosal microbiota metagenome of European eels from five sources (Carda-Diéguez et al., 2014), intestinal microbiota compositions of Japanese eel (Anguilla japonica) (Lee et al., 2015), the compositions of the intestinal microbiota of three eel species (Anguilla japonica, A. marmorata, and $A$. bicolor pacifica) (Hsu et al., 2018), the community composition and complexity of the intestinal microbiota in cultivated European eels during three stages of their lifecycle (Huang et al., 2018), and composition and structure of the intestinal microflora of marbled eels at different growth rates (Lin et al., 2019).

The gut microbiota plays a vital role in the health and growth of fish, and there were some apparent differences in the gut microbiota between fast-growing fish and slow-growing fish individuals (Ringø et al., 1997; Sun et al., 2009). The fast-growing fish might harbor a more favorable gut microbiota, which might contribute to isolate autochthonous probiotics (Sun et al., 2009; Lin et al., 2019). Studied suggested methods of intestinal microbiota determination in fish at different growth rates to identify potential probiotic (Sun et al., 2009; Hsu et al., 2018; Lin et al., 2019). The apparent difference in growth rate existed in the same batch of eels during the cultivation process, which might be caused by the differential gut microbiota under the same culture condition. The present study aims aimed to reveal the difference of intestinal microbiota of European eels with different growth rates by using a next-generation sequencing-based on Illumina technology and provide the basis to identify the autochthonous probiotics for eel's cultivation.

\section{Materials and methods}

\section{Sample collection}

The European eels were cultured synchronously in the indoor concrete tanks with water recirculation system (the area of the hexagonal tank was $30 \mathrm{~m} 2$, water depth was $0.9 \mathrm{~m}$ ) in Fujian Jinjiang Zhiman Aquatic Technology Co., Ltd. (Zhangzhou, China) for one year. The water temperature varied 24 to $30^{\circ} \mathrm{C}$, the $\mathrm{pH}$ varied between 6.0 and 7.5 , the dissolved oxygen concentration is $10-15 \mathrm{mg} / \mathrm{L}$, and the nitrite concentration is $0.15-0.22 \mathrm{mg} / \mathrm{L}$. Fish were fed with commercial power feed (Fujian Tianma Science and Technology Group Co., Ltd., Fuzhou, China).

The diet was mixed with 1.1 volume water to make a dough. The dough was placed on a feeding table and served to the eels. The fish were fed to apparent satiation twice daily (at 6:00 $\mathrm{h}$ 
and 18:00 h). All procedures for culture and sampling of the fish in the present trial were approved by the Chinese Association of Animal Science and Veterinary Medicine and Animal Welfare Committee of Jimei University. Eels were grouped according to the following weight classes: $5-10 \mathrm{~g}$ per fish as slow growth (SG) group, 250-300 g per fish as medium-size growth (MG) group, and 450-500g per fish as fast growth (FG) group. On the final day of the study, the experimental eels were sampled randomly and harvested after $24 \mathrm{~h}$ of fasting, which swam regularly, foraged actively, and appeared disease-free. Then the fish were transported on ice, anesthetized with 200 $\mathrm{mg} / \mathrm{L}$ dose of MS-222 for 10-15 min and rinsed with $75 \%$ ethanol before dissection with sterile scissors. The intestines were separated from the abdominal cavity, and they were flushed with PBS buffer to remove all feces and digested material. Then, the mucosa of the medium intestine of each sample was collected into a sterile $1.5-\mathrm{ml}$ centrifuge tube. Each intestine sample was pooled from three eels. All the samples were labeled as A1-A4 $(n=4)$ for slow growth eels, A4-A8 $(n=4)$ for medium growth eels, and A9-A12 $(n=4)$ for fast growth eels. All specimens were stored and kept frozen at $-80^{\circ} \mathrm{C}$ until DNA extraction.

\section{DNA extraction and PCR amplification}

Total bacterial DNA was extracted from each sample following the instruction of Fast DNA ${ }^{\mathrm{TM}}$ SPIN Kit (MP Biomedicals, CA, USA). The DNA quality was checked by agarose gel electrophoresis and spectrophotometry detection at $260 \mathrm{~nm} / 280 \mathrm{~nm}$. The PCR was carried out on a Mastercycler Gradient (Eppendorf, Germany) using $25 \mu \mathrm{l}$ reaction volumes, containing $12.5 \mu \mathrm{l} 2 \times$ Taq PCR MasterMix, $3 \mu \mathrm{l} \mathrm{BSA}(2 \mathrm{ng} / \mu \mathrm{l}), 2$ Primer ( $5 \mathrm{uM}), 2 \mu \mathrm{l}$ template DNA, and $5.5 \mu \mathrm{lddH} 2 \mathrm{O}$. Further, the V3-V4 hypervariable region of bacterial 16S rDNA gene was amplified using the standard primers fused to barcodes: the forward 338F (5'-ACTCCTACGGGAGGCAGCAG-3') and the reverse 806R (5'GACTACHVGGGTWTCTAAT-3'). The thermal cycling condition of PCR amplification was as followed: initiation at $95^{\circ} \mathrm{C}$ for $5 \mathrm{~min}$, starting 25 cycles at $95^{\circ} \mathrm{C}$ for $30 \mathrm{~s}, 55^{\circ} \mathrm{C}$ for $30 \mathrm{~s}$, and $72{ }^{\circ} \mathrm{C}$ for 30 $\mathrm{s}$, and with a final extension of $72^{\circ} \mathrm{C}$ for $10 \mathrm{~min}$. The amplicon mixture was applied to the MiSeq Genome Sequencer (Illumina, San Diego, CA, USA) by Beijing Allwegene Tech., Ltd. (Beijing, China) (Zheng et al., 2019).

\section{High throughput sequencing}

Deep sequencing was performed on the Miseq platform. The raw data were first screened, and sequences were removed from consideration if they were shorter than $200 \mathrm{bp}$, had a lowquality score $(\leq 20)$, contained ambiguous bases, or did not exactly match to primer sequences and barcode tags. Qualified reads were separated using the sample-specific barcode sequences and trimmed with Illumina Analysis Pipeline Version 2.6. And then, the dataset was analyzed using QIIME. The sequences were clustered into operational taxonomic units (OTUs) at a similarity level of $97 \%$, to calculate the alpha diversity indexes.

\section{Statistical analyses}

The Chao 1 and Shannon index of European eel intestine samples of different groups were calculated with the vegan package (https://www.rdocumentation.org/packages/vegan/versions/ 2.4-2) in R programming for determining bacterial diversity. The results of the OTUs number, Chao1, and Shannon index are presented as means \pm SD. Those data from each treatment group were subjected to a one-way analysis of variance (ANOVA) with SPSS 22.0 statistical software (SPSS, Chicago, IL, USA). When overall differences were significant $(P<0.05)$, Duncan's multiple range test was used to compare the mean values among different treatment groups. Data expressed as percentages or ratios were subjected to arcsine transformation before statistical analysis. The relative abundance of intestinal microbiota at the genus level was subjected to Kruskal-Wallis test with qvalue package (https://www.rdocumentation.org/packages/qvalue/ versions/2.4.2) of $\mathrm{R}$ programming for determining bacterial differences, and the significant level was $\mathrm{P}<0.05$. 


\section{Results}

The alpha diversity indexes of the intestinal microbiota of European eels

The Rarefaction curves of all the samples are shown in figure 1 . The tendency of the curves to plateau showed that the amount of sequencing data was sufficient to reflect the vast majority of microbial information in the sample.

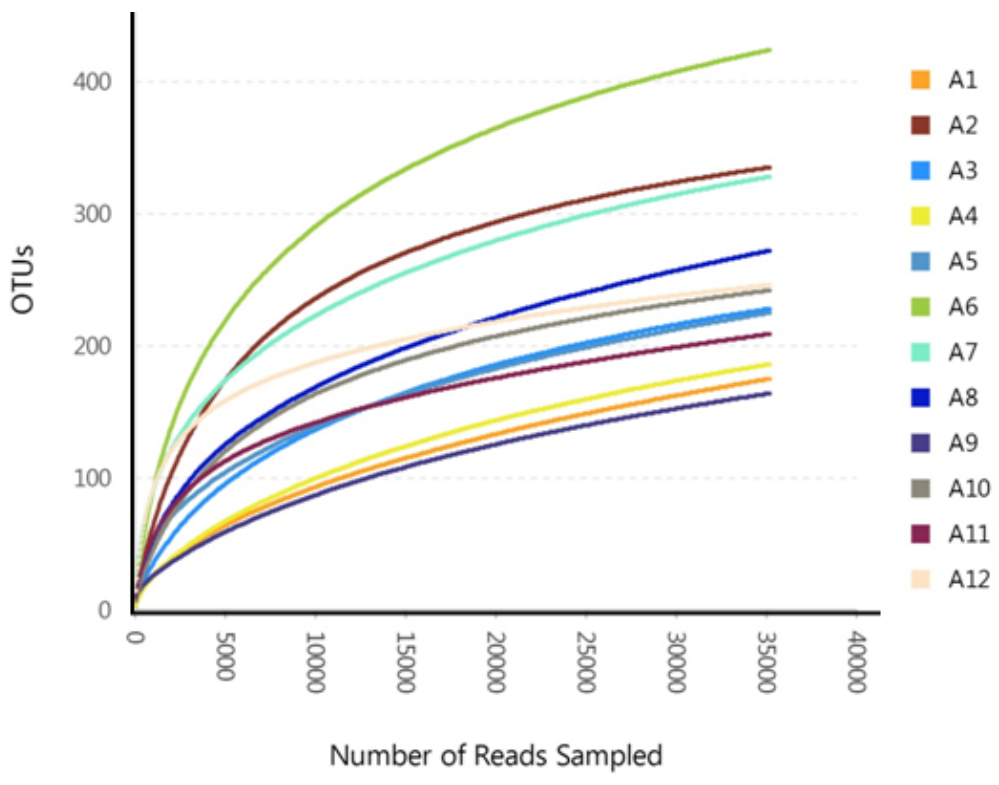

Figure 1 Rarefaction curve of all intestinal samples of European eels

The intestinal samples of A1-A4 from European eels in a slow-growth group, A5-A8 samples from European eels in the medium growth group, A9-A12 samples from European eels in the fastgrowing group. The alpha diversity indexes of the intestinal microbiota of European eels are presented in Table 1.

Table 1 Bacterial diversity index analysis estimated sample coverage based on 16S rDNA gene sequences of intestine samples of European eels

\begin{tabular}{cccc}
\hline Item & SG group & MG group & FG group \\
\hline OTUs & $231 \pm 80 \mathrm{a}$ & $312 \pm 86 \mathrm{a}$ & $215 \pm 38 \mathrm{a}$ \\
Chao1 & $307.59 \pm 50.90 \mathrm{a}$ & $399.18 \pm 80.05 \mathrm{~b}$ & $281.76 \pm 18.72 \mathrm{a}$ \\
Shannon & $1.09 \pm 0.42 \mathrm{a}$ & $2.63 \pm 0.98 \mathrm{~b}$ & $2.67 \pm 0.81 \mathrm{~b}$ \\
Coverage & $0.9976 \pm 0.0126 \mathrm{a}$ & $0.9975 \pm 0.0350 \mathrm{a}$ & $0.9980 \pm 0.0287 \mathrm{a}$ \\
\hline
\end{tabular}

\footnotetext{
${ }^{\mathrm{ab}}$ Values within the same line without the same superscript were significantly different at $\mathrm{P}<0.05$ level. $\mathrm{SG}=$ slow growth, $\mathrm{MG}=$ medium growth, $\mathrm{FG}=$ fast growth .
}

The value of the Chao 1 index showed that bacteria richness was significantly higher in the MG group in comparison with the SG group and the FG group $(P<0.05)$, and there was no significant difference in the above indexes between the SG group and the FG group $(P>0.05)$. The values of the Shannon index showed that bacteria diversity was increased significantly in the MG group and the FG group in comparison with the SG group $(P<0.05)$, and there was no significant difference in the above indexes between the MG group and the FG group $(P>0.05)$. The differences between OUTs and coverage values were not significant among all the three groups $(P>0.05)$. 
The change of Intestinal microbiota composition at the phylum level of European eels

The change of intestinal microbiota composition at the phylum level of European eels with different growth rates was presented in figure 2 .

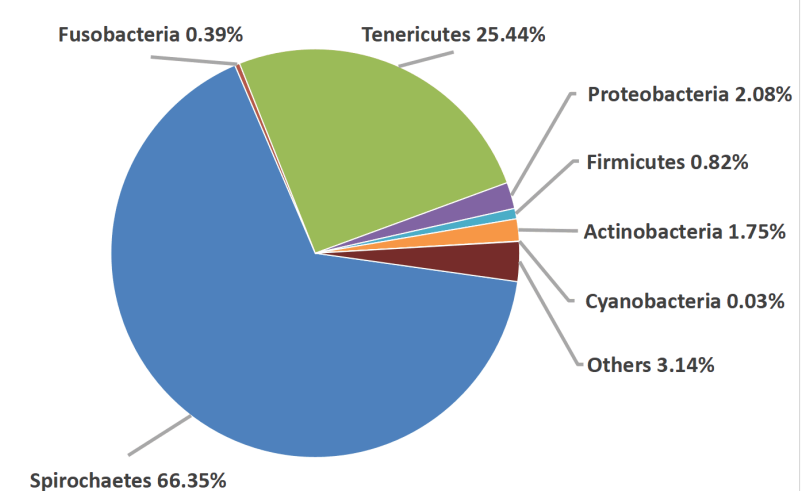

SG

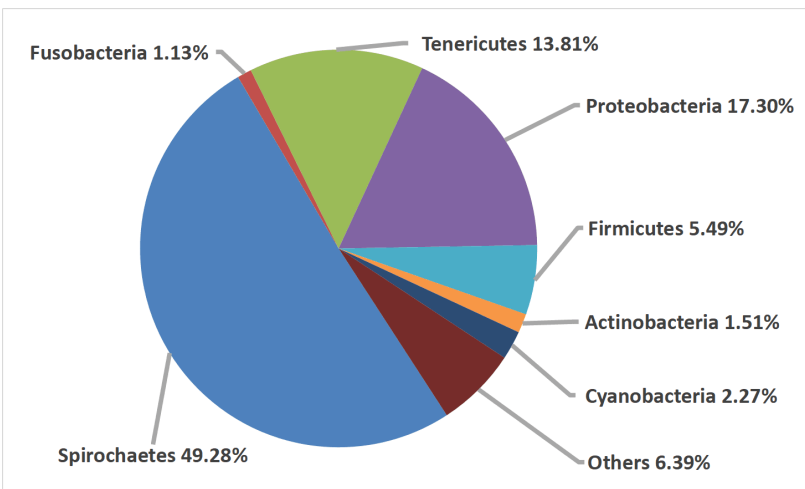

MG

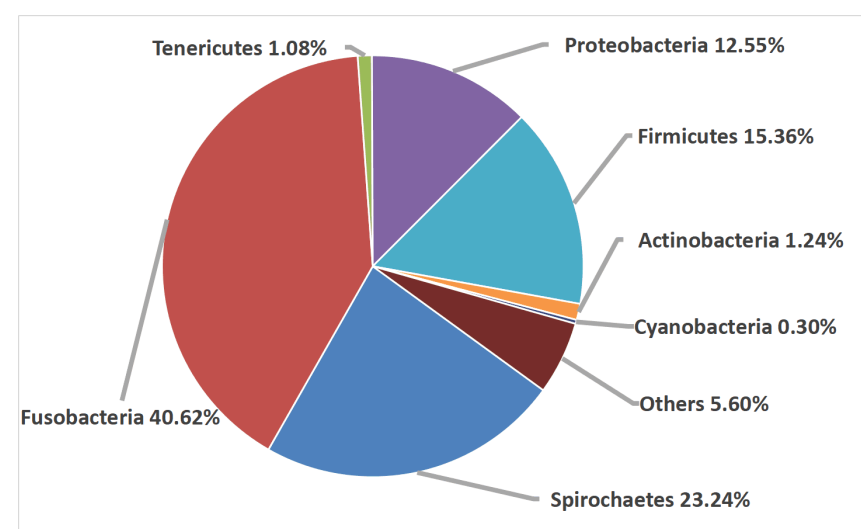

FG

Figure 2 The intestinal microbiota composition at phylum level of European eel with a different growth rate $\mathrm{SG}=$ slow growth group, $\mathrm{MG}=$ medium growth group, $\mathrm{FG}=$ fast growth group

The predominant phylum of European eels in the SG group and MG group were Spirochaetes at the phylum level of intestinal microbiota, while Fusobacteria was predominant phylum of European eels in FG group. With the increasing growth rate, there was an apparent decreasing trend of relative abundance of Spirochaetes and Tenericute, and the relative abundances of Fusobacteria, Proteobacteria, and Firmicutes showed an increasing trend. Compared with the SG group, the European eels in the FG group had the higher relative abundances of Fusobacteria, Proteobacteria, and Firmicutes and lowered relative abundances of Spirochaetes and Tenericute. The change of intestinal microbiota based on genus level of European eel with different growth rates. The statistically significant change of the intestinal microbiota at the genus level of European eel with different growth rates was showed in figure 3 and figure 4. 


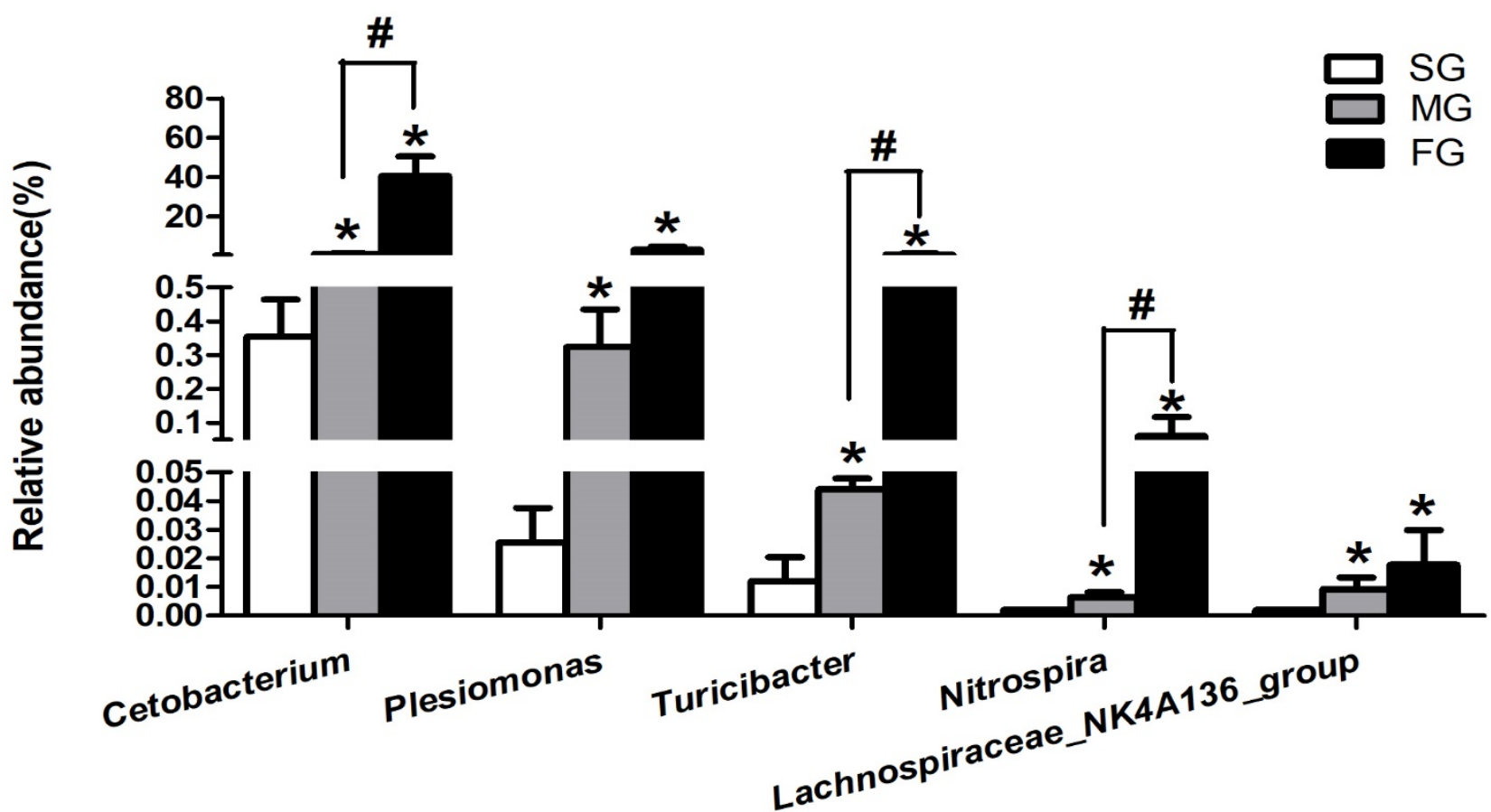

Figure 3 The intestinal microbiota with the increasing trend of relative abundance at the genus level of European eels with a different growth rate $\mathrm{SG}=$ slow growth group, $\mathrm{MG}=$ medium growth group, $\mathrm{FG}$ =fast growth group.

* above the bars indicated that there was a significant difference between related groups and SG group at $\mathrm{P}<0.05$ level.

\# above the bars indicated that there was a significant difference between MG groups and the FG group at $\mathrm{P}<0.05$ level.

The relative abundances of Cetobacterium, Plesiomonas, Turicibacter Nitrospira, and Lachnospiraceae bacterium NK4A136 group increased with the increasing growth rate. The relative abundances of the above bacteria SG group were significantly lower than those in the other two groups $(P<0.05)$. There were significant differences in relative abundances of Cetobacterium, Turicibacter, and Nitrospira between the MG group and the FG group $(P<0.05)$, and the differences of relative abundance of Plesiomonas and Lachnospiraceae bacterium NK4A136 group were not significant between those two groups $(P>0.05)$. 


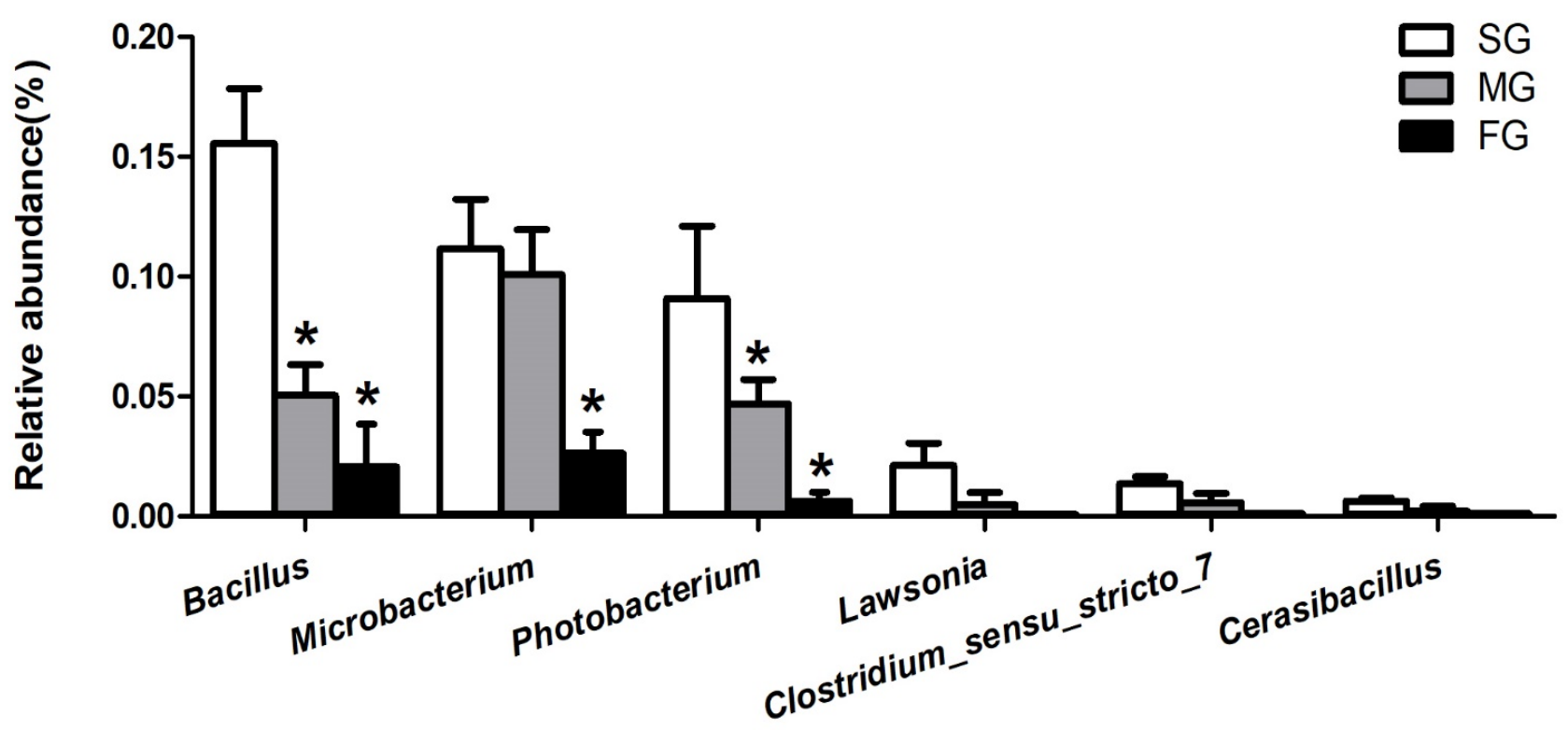

Figure 4 The intestinal microbiota with the decreasing trend of relative abundance at the genus level of European eels with a different growth rate

$\mathrm{SG}$ =slow growth group, $\mathrm{MG}=$ medium growth group, $\mathrm{FG}$ =fast growth group.

* above the bars indicated that there was a significant difference between related groups and SG group at $\mathrm{P}<0.05$ level.

\# above the bars indicated that there was a significant difference between MG groups and the FG group at $\mathrm{P}<0.05$ level.

The relative abundances of Bacillus, Microbacterium, and Photobacterium in the intestine of European eels of FG group were significantly lower than those in SG group $(P<0.05)$, there were no significant differences of those bacteria (except Microbacterium) between FG group and MG group $(P>0.05)$. The differences of the relative abundance of the above bacteria (except Bacillus and Photobacterium) were not significant between the MG group and SG group $(P>0.05)$. The Lawsonia, Clostridium sensustricto 7, and Cerasibacillus were not observed in the FG group.

\section{Discussion}

The change of Bacterial richness and diversity indexes in the intestine of European eels with different growth rates

The number of OTUs and Chao1 was usually employed to estimate the total number of species, which represent the richness of bacteria species in an ecosystem. Higher values of the Shannon index indicate greater diversity of bacteria species. The coverage index refers to the coverage rate of the sample library; a higher value indicates a lower probability that a sample was not measured (Huang et al., 2018; Lin et al., 2019).

The number of OTUs found in the intestine of eels in all three groups was not different statically. The indexes of Chao1 and Shannon of the MG group were higher than those of the SG group, which indicated that the European eels with a faster growth rate might possess higher microbial richness and diversity. chao1 in the FG group was similar in the SG group, while the Shannon of the FG group was higher than that of the SG group. These results were consistent with the report of Lin et al. (2019), they found that the fast growth marbled eels had similar OTUs, Chao 1 and Shannon indexes with the stunted growth eels, and Chao1 index of medium growth marbled eels was higher than that of stunted growth eel. Besides, the intestine samples of the European eel at the silver stage showed significantly higher species richness estimated by Chao1 than the ones at the yellow eel stage (Huang et al., 2018). The higher species richness of gut 
microbiota was also found in more significant size individuals of mangrove killifish (Kryptolebias marmoratus) and Atlantic cod (Gadus morhua) (Forberg et al., 2016) and fast-growing grouper (Epinephelus coioides) (Sun et al., 2009). Compared with the slow growth of yellow croaker (Pseudosciaena crocea), the typical growth fish had lower Chao 1 and similar Shannon index (Li et al., 2017). The intestinal microbiota of the adult European eels had lower bacterial diversity and fewer phylotypes than those of younger elvers (Huang et al., 2018). The different changes of intestinal microbiota alpha diversity indexes of different fish species might be related to the genetic background and rearing environment except for the growth rate (Giatsis et al.,2015; Forberg et la., 2016; Hsu et al., 2018). The average value of these indexes was ranged from $99.75 \%$ to $99.80 \%$ in the present study, which could reflect a reliable analysis of the operational taxonomic units (OTUs) detected in the sample.

The change of intestine microbiota composition at phylum level of European eel with different growth rates

Fusobacteria is the dominant phylum in the European eels of the FG group, which is consistent with the previous study of marbled eel with a fast growth rate (Lin et al., 2019). Fusobacteria were regarded as dominant members of the gut microbiota of freshwater fishes. It is anaerobic, Gram-negative bacilli that produce butyrate, a short-chain fatty acid that is often the end product of the fermentation of carbohydrates (Larsen et al., 2014). some bacteria in Fusobacterium can synthesize multiple vitamins, elicit host proinflammatory response and possess virulence characteristics that promote their adhesiveness to host epithelial cells and their ability to invade into epithelial cells (Kostic et al., 2012). In the present study, the proportion of Firmicutes and Proteobacteria in the European eels of the MG group and FG group was higher than those of the SG group. Similar results were reported in the research of comparison of intestinal microbiota composition at the phylum level between fast growth eels and stunted growth eels (Lin et al., 2019). Firmicutes and Proteobacteria were considered to produce protease (da Cruz Ramos et al., 2016). The Firmicutes might assist in the nutritional processes of complex and undigested polysaccharides ( $\mathrm{Ni}$ et al., 2014), stimulate the absorption and metabolism of fatty acids in the fish gut, and promote host energy absorption and storage together (Zhou et al., 2018). Besides, many genera of lactic acid bacteria belonging to Firmicutes, constitute a normal part of the intestinal microbiota of fish and are generally considered beneficial microorganisms associated with a healthy intestinal epithelium (Hovda et al., 2007; Roeselers et al.,2011). Proteobacteria members have been reported to decompose chemical compounds that can serve as a source of energy and metabolites, and improve the health status of fish (Nyman et al., 2017). So many beneficial effects of Fusobacteria, Firmicutes, and Proteobacteria with higher relative abundance might contribute to European eels to get a fast growth rate.

The proportion of Spirochaetes and Tenericute were lowest in eels' intestine of the FG group. Spirochaetes accounted for very little of the microbiota composition in most of the fish (Radolf et al., 2012), and they might involve with the disease of some aquatic animals (Matsuyama et al., 2017). A previous study indicated that Tenericutes was increased significantly in the guts of stunted-growth marbled eels (Lin et al., 2019), which was consistent with the present study. The decreasing proportion of Spirochaetes and Tenericute in the intestine might be beneficial to the gut health of European eels.

\section{The difference of intestine microbiota at the genus level of European eel with different growth rates}

In the present research, the European eels with a fast growth rate had higher relative abundances of Cetobacterium, Plesiomonas, Turicibacter Nitrospira, and Lachnospiraceae bacterium NK4A136 group in comparison with slow-growth ones. A similar change of Cetobacterium was reported in the study of the composition and structure of the intestinal microbiota of marbled eels at different growth rates (Lin et al., 2019). Huang et al. (2018) also found that the Cetobacterium in the European yellow eels was significantly higher than that of silver eels and elver eels. Cetobacterium and particularly C. somerae, are known to synthesize cobalamin in fish without dietary sources of the vitamin, signifying a compensatory enrichment of the taxa in response to either malabsorption of the vitamin, or its deficiency in the diet (Koo et al., 
2017). Cetobacterium was also found to produce vitamin B12 efficiently and ferment peptides and sugars, which were closely related to the nutrition of fish. Also, Cetobacterium could synthesize fats, proteins, and carbohydrates used by the host and play an essential role in growth and development (Hsu et al., 2018). Thus, the higher abundance of Cetobacterium presented in the intestine of fast growth farmed European eels may play an essential role in the nutrient metabolism and growth promotion effect, which indicated that it might be the potential probiotics for the European eels.

Turicibacter was commonly found in the gut of freshwater fish species and other animals (Kamada et al., 2013; Ray et al., 2012), but its origin and role were still unclear. Plesiomonas were reported as the potential opportunistic pathogens in the fish intestine, which may have detrimental impacts on fish health (Pakingking et al.,2015; Wang et al., 2019). Although the relative abundance of Plesiomonas was higher in eels of the FG group, no intestine health problem was observed in the sampling process and cultivation. Nitrospira was primarily responsible for the oxidation of nitrate, which was also observed in the other fish intestine (Kashinskaya et al., 2015). The presence of the Lachnospiraceae bacterium NK4A136 group was negatively correlated with intestinal inflammation (Zhao et al., 2017). The Nitrospira and Lachnospiraceae bacterium NK4A136 group were not observed in the eels of the SG group; those two bacteria might have a beneficial effect on the intestine health of eels. This point should be confirmed via further studies.

In the present research, the European eels with a fast growth rate had lower relative abundances of Bacillus, Microbacterium, and Photobacterium in comparison with slow growth eels. Some probiotic Bacillus has been reported as fish growth promoters (Liu et al., 2012), while many studies using Bacillus showed no beneficial effect on the host (Ran et al., 2012). From the decreasing trend of relative abundance of Bacillus in eels' intestine of the FG group, we could infer that Bacillus might have no beneficial effects on the intestinal health of European eels. Microbacterium were known for their ability to produce antimicrobial compounds, and negatively correlated with the development of enteritis (Navarrete et al., 2013); its relative abundance was decreased in the fast growth eels, no enteritis was observed in the intestine of all eels. Some photo bacteria of Photobacterium are symbiotic or pathogenic for fish and crustaceans, and they were classified as an emerging pathogen in fish culture (Labella et al., 2017).

The Lawsonia, Clostridium sensu stricto 7, and Cerasibacillus were not observed in fastgrowth eels. Lawsonia was previously found at very low abundance in conventionally raised zebrafish (Roeselers et al., 2011), and might be the opportunistic microbes (Earley et al., 2018). Clostridium has been described as producers of volatile fatty acids, vitamins, and digestive enzymes in the fish intestine (Ray et al., 2012). The relative abundance of Clostridium sensu stricto 7 in the SG group and the MG group might have little effect on the digestion and absorption of feed nutrients in the fish gut. At present, Cerasibacillus was seldom reported in fish, and its function in the intestine should be clarified in future research. It was firstly reported to play an essential role in the degradation of organic materials and isolated from a semi-continuous decomposing system of kitchen refuse (Nakamura et al., 2004). More studies are needed to reveal the function of those three bacteria in European eels' intestine.

In conclusion, this study presented the first report to investigate the composition, diversity, and abundance of intestinal microbiota among European eels with different growth rates by using next-generation sequencing technology. Our results indicated that the intestinal microbiota composition was affected by the growth rate of European eels; significant differences were observed between fast growth eels and slow growth eels. The relative abundance of some bacteria in intestinal microbiota were significantly different for the European eels with different growth rates. The Cetobacterium seemed to be great potential probiotics from the change of differential bacteria between fast growth eels and slow growth eels. We hoped that more studies could be conducted to accelerate the practical application of the candidate probiotics to eel farming.

\section{Acknowledgment}

This study was supported by the China Agriculture Research System (CARS-46), Key Science and Technology Project of Fujian Province (2018N0020), and Innovative Training Program for College Student of Jimei University(2019xj104). 


\section{References}

Carda-Diéguez M., Ghai R., Rodriguez-Valera F. and C. Amaro, 2014. Metagenomics of the mucosal microbiota of European eels. Genome Announc., 2(6):e01132.

da Cruz Ramos G.F., Ramos P.L., Passarini M.R.Z., Silveira M.A.V., Okamoto D.N., de Oliveira L.C.G., Zezzo L.V., Marem A., Rocha R.C.S., da Cruz J.B., Juliano L. and SP de Vasconcellos, 2016. Cellulolytic and proteolytic ability of bacteria isolated from gastrointestinal tract and composting of a hippopotamus. AMB Express, 6:17.

Earley A.M., Graves C.L. and C.E. Shiau, 2018. Critical role for a subset of intestinal macrophages in shaping gut microbiota in adult zebrafish. Cell Rep., 25(2):424-436.

Forberg T., Sjulstad E.B., Bakke I., Olsen Y., Hagiwara A., Sakakura Y. and O. Vadstein, 2016. Correlation between microbiota and growth in Mangrove Killifish (Kryptolebias marmoratus) and Atlantic cod (Gadus morhua). Sci. Rep., 6:21192.

Giatsis C., Sipkema D., Smidt H., Heilig H., Benvenuti G., Verreth J. and M. Verdegem, 2015. The impact of rearing environment on the development of gut microbiota in tilapia larvae. Sci. Rep., 5:18206.

Hoseinifar S.H., Sun Y., Wang A. and Z. Zhou, 2018. Probiotics as a means of disease control in aquaculture, a review of current knowledge and future perspectives. Front Microbiol., 9:2429.

Hovda M.B., Lunestad B.T., Fontanillas R. and J.T. Rosnes, 2007. Molecular characterization of the intestinal microbiota of farmed Atlantic salmon (Salmo salar L.). Aquaculture, 272(1-4):581588.

Hsu H.Y., Chang F.C., Wang Y.B., Chen S.H., Lin Y.P., Lin C.Y. and Y.S. Han, 2018. Revealing the compositions of the intestinal microbiota of three Anguillid eel species using 16S rDNA sequencing. Aquac. Res., 49(7):2404-2415.

Huang W., Cheng Z., Lei S., Liu L., Lv X., Chen L., Wu M., Wang C., Tian B. and Y. Song, 2018. Community composition, diversity, and metabolism of intestinal microbiota in cultivated European eel (Anguilla anguilla). Appl. Microbiol. Biot., 102(9):4143-4157.

Jacoby D.M.P., Casselman J.M., Crook V., DeLucia M., Ahn H., Kaifu K., Kurwie T., Sasal P., Silfvergrip A.M.C., Smith K.G., Uchida K., Walker A.M. and M.J. Gollock, 2015. Synergistic patterns of threat and the challenges facing global anguillid eel conservation. Glob. Ecol. Conserv., 4:321-333.

Kamada N., Chen G.Y., Inohara N. and G. Núnez, 2013. Control of pathogens and pathobionts by the gut microbiota. Nat. Immunol., 14(7):685-690.

Kashinskaya E.N., Belkova N.L., Izvekova G.I., Simonov E.P., Andree K.B., Glupov V.V., Baturina O.A., Kabilov M.R. and MM Solovyev, 2015. A comparative study on microbiota from the intestine of Prussian carp (Carassius gibelio) and their aquatic environmental compartments, using different molecular methods. J. Appl. Microbiol., 119(4):948-961.

Koo H., Hakim J.A., Powell M.L., Kumar R., Eipers P.G., Morrow C.D., Crowley M., Lefkowitz E.J., Watts S.A. and A.K. Bej, 2017. Metagenomics approach to the study of the gut microbiome structure and function in zebrafish Danio rerio fed with gluten formulated diet. J. Microbiol. Meth., 135:69-76.

Kostic A.D., Gevers D., Pedamallu C.S., Michaud M., Duke F., Earl A.M., Ojesina A.I., Jung J., Bass A.J., Tabernero J., Baselga J., Liu C., Shivdasani R.A., Ogino S., Birren B.W., Huttenhower C., Garrett W.S. and M. Meyerson, 2012. Genomic analysis identifies association of Fusobacterium with colorectal carcinoma. Genome Res., 22(2):292-298.

Labella A.M., Arahal D.R., Lucena T., Manchado M., Castro D. and J.J. Borrego, 2017. Photobacterium toruni sp. nov., a bacterium isolated from diseased farmed fish. Int. J. Syst. Evol. Micr., 67(11):4518-4525.

Larsen A.M., Mohammed H.H., Arias C.R., 2014. Characterization of the gut microbiota of three commercially valuable warm-water fish species. J. Appl. Microbiol., 116(6): 1396-1404.

Lee J.S., Damte D., Lee S.J., Hossain M.A., Belew S., Kim J.Y., Rhee, M.H., Kim, J.C., and SC. Park, 2015. Evaluation and characterization of a novel probiotic Lactobacillus pentosus PL11 isolated from Japanese eel (Anguilla japonica) for its use in aquaculture. Aquacult. Nutr., 21(4):444-456.

Li Y.Y., Chen X. and TY. Song, 2017. Differences in intestinal flora of cultured large yellow croaker Pseudosciaena crocea with different growth rates. J. Dalian Ocean Univ., 32(5):509-513. 
Lin M., Zeng C.X., Jia X.Q., Zhai SW, Li ZQ. and Y. Ma, 2019. The composition and structure of the intestinal microflora of Anguilla marmorata at different growth rates: a deep sequencing study. J. Appl. Microbiol., 126(5):1340-1352.

Liu C.H., Chiu C.H., Wang S.W. and W.T. Cheng, 2012. Dietary administration of the probiotic, Bacillus subtilis E20, enhances the growth, innate immune responses, and disease resistance of the grouper, Epinephelus coioides. Fish Shellfish Immun., 33(4):699-706.

Matsuyama T., Yasuike M., Fujiwara A., Nakamura Y., Takano T., Takeuchi T., Satoh N., Adachi Y., Tsuchihashi Y., Aoki H., Odawara K., Iwanaga S., Kurita J., Kamaishi T. and C. Nakayasu, 2017. A Spirochaete is suggested as the causative agent of Akoya oyster disease by metagenomic analysis. PLoS One, 12(8):e0182280.

Mladineo I., Bušelić I., Hrabar J., Radonić I., Vrbatović A., Jozić S. and Ž. Trumbić, 2016. Autochthonous bacterial isolates successfully stimulate in vitro peripheral blood leukocytes of the European sea bass (Dicentrarchus labrax). Front Microbiol., 7:1244.

Mordenti O., Di Biase A., Sirri R., Modugno S. and A. Tasselli, 2012. Induction of sexual maturation in wild female European eel (Anguilla anguilla) in darkness and light conditions. Isr. J. Aquacult-Bamidgeh, IJA_64.2012.726,1-9 pages.

Nakamura K., Haruta S., Ueno S., Ishii M., Yokota A. and Y. Igarashi, 2004. Cerasibacillus quisquiliarum gen. nov., sp. nov., isolated from a semi-continuous decomposing system of kitchen refuse. Int. J. Syst. Evol. Micr., 54(4):1063-1069.

Navarrete P., Fuentes P., De la Fuente L., Barros L., Magne F., Opazo R., Ibacache C., Espejo R. and J. Romero, 2013. Short-term effects of dietary soybean meal and lactic acid bacteria on the intestinal morphology and microbiota of Atlantic salmon (Salmo salar). Aquacult. Nutr., 19(5):827-836.

Ni J., Yan Q., Yu Y. and T. Zhang, 2014. Factors influencing the grass carp gut microbiome and its effect on metabolism. FEMS Microbiol. Ecol., 87(3):704-714.

Nyman A., Huyben D., Lundh T. and J. Dicksved, 2017. Effects of microbe-and mussel-based diets on the gut microbiota in Arctic charr (Salvelinus alpinus). Aquacult. Rep., 5:34-40.

Pakingking R., Palma P. and R. Usero, 2015. Quantitative and qualitative analyses of the bacterial microbiota of tilapia Oreochromis niloticus cultured in earthen ponds in the Philippines. World J. Microb. Biot., 31(2):265-275.

Radolf J.D., Caimano M.J., Stevenson B. and L.T. Hu, 2012. Of ticks, mice and men: Understanding the dual-host lifestyle of Lyme disease Spirochaetes. Nat. Rev. Microbiol., 10(2):87-99.

Ran C., Carrias A., Williams M.A., Capps N., Dan B.C.T., Newton J.C., Kloepper J.W., Ooi E.L., Browdy C.L., Terhune J.S. and M.R. Liles, 2012. Identification of Bacillus strains for biological control of catfish pathogens. PLoS One, 7(9):e45793.

Ray A.K., Ghosh K. and E. Ringø, 2012. Enzyme-producing bacteria isolated from fish gut: A review. Aquac. Nutr., 18(5):465-492.

Ringø E., Olsen R.E., Øverli Ø. and F. Løvik, 1997. Effect of dominance hierarchy formation on aerobic microbiota associated with epithelial mucosa of subordinate and dominant individuals of Arctic charr, Salvelinus alpinus (L.). Aquac. Res., 28(11): 901-904.

Roeselers G., Mittge E.K., Stephens W.Z., Parichy D.M., Cavanaugh C.M., Guillemin K. and J.F. Rawls, 2011. Evidence for a core gut microbiota in the zebrafish. ISME J., 5(10):1595-1608. Sun Y.Z., Yang H.L., Ling Z.C., Chang J.B. and J.D. Ye, 2009. Gut microbiota of fast and slowgrowing grouper Epinephelus coioides. Afr. J. Microbio. Res., 3(11):713-720.

Wang E.L., Yuan Z.H., Wang K.Y., Gao D.Y., Liu Z.J. and M.R. Liles, 2019. Consumption of florfenicol-medicated feed alters the composition of the channel catfish intestinal microbiota including enriching the relative abundance of opportunistic pathogens. Aquaculture, 501:111-118. Zhao L., Zhang Q., Ma WN, Tian F., Shen H.Y. and M.M. Zhou, 2017. A combination of quercetin and resveratrol reduces obesity in high-fat diet-fed rats by modulation of gut microbiota. Food Funct., 8(12):4644-4656.

Zheng J.P., Yuan X.B., Zhang C., Jia P.Y., Jiao S.M., Zhao X.M., Yin H., Du Y.G. and H.T. Liu, 2019. N-Acetylcysteine alleviates gut dysbiosis and glucose metabolic disorder in high-fat dietfed mice. J. diabetes, 11(1): 32-45. 
Zhou M., Liang R.S., Mo J.F., Yang S., Gu N., Wu Z.H., Babu S.V., Li J., Huang Y.M. and L. Lin, 2018. Effects of brewer's yeast hydrolysate on the growth performance and the intestinal bacterial diversity of largemouth bass (Micropterus salmoides). Aquaculture, 484:139-144. 